\title{
RNAi therapy targeting KRAS in combination with chemotherapy for locally advanced pancreatic cancer patients
}

\author{
Talia Golan¹, Elina Zorde Khvalevsky², Ayala Hubert ${ }^{3}$, Rachel Malka Gabai², Naama \\ Hen $^{2}$, Amiel Segal ${ }^{4}$, Abraham Domb ${ }^{5}$, Gil Harari ${ }^{6}$, Eliel Ben David7, Stephen Raskin ${ }^{1}$, \\ Yuri Goldes ${ }^{1}$, Eran Goldin ${ }^{8}$, Rami Eliakim¹, Maor Lahav ${ }^{1}$, Yael Kopleman ${ }^{9}$, Alain \\ Dancour $^{8}$, Amotz Shemi ${ }^{2}$ and Eithan Galun ${ }^{10}$ \\ ${ }^{1}$ The Sackler School of Medicine, The Chaim Sheba Medical Center, Tel Aviv University, Tel Aviv, Israel \\ 2 Silenseed Ltd., Jerusalem, Israel \\ ${ }^{3}$ Sharett Institute of Oncology, Hadassah-Hebrew University Medical Center, Jerusalem, Israel \\ ${ }^{4}$ The Gastroenterology Institute, Shaare Zedek Medical Centre, Jerusalem, Israel \\ ${ }^{5}$ Institute of Drug Research, School of Pharmacy-Faculty of Medicine, Center for Nanoscience and Nanotechnology and The \\ Alex Grass Centre for Drug Design and Synthesis, The Hebrew University of Jerusalem, Jerusalem, Israel \\ ${ }^{6}$ MediStat Ltd., Jerusalem, Israel \\ 7 Hadassah-Hebrew University Medical Centre, Jerusalem, Israel \\ ${ }^{8}$ The Gastroenterology Institute, Shaare Zedek Medical Center, Jerusalem, Israel \\ ${ }^{9}$ Gastroenterology Institute, Hadassah Hebrew University Medical Center, Jerusalem, Israel \\ 10 The Goldyne Savad Institute for Gene Therapy, Hadassah Hebrew University Medical Center, Jerusalem, Israel \\ Correspondence to: Talia Golan, email: Talia.Golan@sheba.health.gov.il \\ Keywords: Pancreatic cancer, KRAS, overall survival, siRNA, polymer implant \\ Received: March 04, 2015 \\ Accepted: May 02, 2015 \\ Published: May 19, 2015
}

This is an open-access article distributed under the terms of the Creative Commons Attribution License, which permits unrestricted use, distribution, and reproduction in any medium, provided the original author and source are credited.

ABSTRACT

Purpose: The miniature biodegradable implant siG12D-LODER ${ }^{\mathrm{TM}}$ was inserted into a tumor and released a siRNA drug against KRAS(G12D) along four months. This novel siRNA based drug was studied, in combination with chemotherapy, as targeted therapy for Locally Advanced Pancreatic Cancer (LAPC).

Methods: An open-label Phase 1/2a study in the first-line setting of patients with non-operable LAPC was initiated. In this study patients were assigned to receive a single dose of siG12D-LODERs, in three escalating dose cohorts $(0.025 \mathrm{mg}, 0.75 \mathrm{mg}$ and $3.0 \mathrm{mg}$ ). Gemcitabine was given on a weekly basis, following the siG12D-LODERTM insertion, until disease progression. The recommended dose was further examined with modified FOLFIRINOX. The follow up period was eight weeks and survival until death.

Results: Fifteen patients with LAPC were enrolled. Among the 15 treated patients, the most frequent adverse events observed were grade 1or 2 in severity ( $89 \%)$; five patients experienced serious adverse events (SAEs). In 12 patients analyzed by CT scans, none showed tumor progression, the majority $(10 / 12)$ demonstrated stable disease and two showed partial response. Decrease in tumor marker CA19-9 was observed in $70 \%(7 / 10)$ of patients. Median overall survival was 15.12 months; 18 month survival was $38.5 \%$.

Conclusions: The combination of siG12D-LODER ${ }^{\mathrm{TM}}$ and chemotherapy is well tolerated, safe and demonstrated a potential efficacy in patients with LAPC. NCT01188785 


\section{INTRODUCTION}

\section{Pancreatic cancer}

Pancreatic ductal adenocarcinoma (PDAC) is the most common pancreatic neoplasm, responsible for $90 \%$ of pancreatic cancer cases [1]. Until recently Gemcitabine was considered the main treatment option. Recent progress in the treatment of metastatic PDAC has been shown with the addition of nab-paclitaxel to Gemcitabine [2] and the introduction of FOLFIRINOX (Leucovorin, Fluorouracil, Irinotecan and Oxaliplatin) [3]. FOLFIRINOX substantially improves overall survival (OS) compared to Gemcitabine, however, it has a high toxicity profile and hence only suitable for a subset of patients. About $80 \%$ to $85 \%$ of patients with pancreatic cancer have advanced disease at the time of diagnosis, i.e. stage III (LAPC) or stage IV (metastatic), and are not candidates for surgical curative intervention. Several autopsy series demonstrated that $30 \%$ of patients presenting with stage III disease died without evidence of distant metastases [4].

Treatment options for LAPC include several chemotherapy regimens (Gemcitabine, Gemcitabine and Erlotinib, Gemcitabine and Nab-Paclitaxel, or FOLFIRINOX) with or without radiation. It is still unclear whether chemoradiation for LAPC patients is warranted. Chauffert et al studied initial chemoradiotherapy (intermittent Cisplatin and infusional 5-FU) followed by Gemcitabine vs. Gemcitabine alone in patients with LAPC and observed a median survival of 8.6 months for the chemoradiation arm vs. 13 months for chemotherapy alone [5]. Loehrer et al. showed that treatment of patients with Gemcitabine alone or with radiation led to survival of 9.2 and 11.1 months respectively [6]. Herman et al. demonstrated that treatment of LAPC patients with chemoradiotherapy (50.4 Gy with concurrent fluorouracil), with or without TNFerade, led to median survival of 10.0 months in both treatment arms. Eighteen month survival was $13.3 \%$ (standard of care (SOC)) vs. $18.2 \%$ (SOC+TNFerade) [7]. Progression Free Survival (PFS) was 7.0 months (SOC) vs. 6.8 months (SOC+TNFerade) and 18 month PFS was 6.6\% (SOC) vs. $8 \%$ (SOC+TNFerade) [7]. Blazer et al. reported that nonresectable FOLFIRINOX-treated patients showed median OS and PFS of 12.7 and 8 months respectively [8].

Epithelial carcinogenesis is typically associated with the accumulation of mutations and genetic lesions, leading to the activation of oncogenes and inactivation of tumor suppressor genes. Mutated KRAS was proposed to be a hallmark of pancreatic cancer [9] since this protein is mutated in more than $90 \%$ of PDACs [10]. Mounting literature data suggest that the KRAS mutations may be early events in the molecular pathological cascade leading to PDAC [11]. The vast majority of KRAS mutations in adenocarcinoma of the human pancreas are gain-offunction mutations, in which most of these occur in codon 12. Among the codon 12 mutations the most abundant is substitution of the Glycine for Aspartate (G12D) [9, 12, 13]. In PDAC cancer cells are addicted to expression of the mutated KRAS [9, 14]. Still, more than 30 years of research have not yielded a specific therapy directly targeting mutated KRAS. The challenges are attributed to the picomolar affinity of this oncogene for GTP/GDP [15, $16]$ and failure to target any relevant allosteric regulatory sites. Recently, the maturing field of mRNA targeting by RNA interference (RNAi) has proven to be a highly potent alternative compared to the more general protein inhibition approach. It was previously shown that suppression of KRAS expression by RNAi led to growth inhibition of PDAC cells in vitro and PDAC-originated tumors in vivo in mice $[17,18]$.

\section{RNAi-based anti-mutated KRAS treatment for patients with LAPC}

To address the unmet need for an efficient nontoxic treatment for patients with LAPC, and to convert the potential of RNAi into an anti-cancer therapy, Silenseed Ltd. has developed the LODER ${ }^{T M}$ (Local Drug EluteR). $L O D E R^{T M}$ presents a novel solution to the major challenges of oligonucleotide therapeutics and RNAi for a large number of diseases including solid tumors. Such challenges include delivery of RNAi-based drugs and achievement of prolonged activity at a tolerated dose at the target site. The siG12D-LODER ${ }^{T M}$ is a miniature biodegradable polymeric matrix that encompasses antiKRASG12D siRNA (siG12D). siG12D-LODER ${ }^{T M}$ is designed to provide a slow and prolonged local drug release within the tumor over several months, while ensuring protection of the siRNA drug from degradation. It can be inserted and placed into pancreatic tumor using a standard endoscope ultrasound (EUS) biopsy procedure. Here, we present the use of siG12D-LODER ${ }^{T M}$ as a new therapeutic modality targeting mutated KRAS in combination with the currently available treatments including Gemcitabine or FOLFIRINOX.

Our treatment of LAPC with siG12D-LODER ${ }^{T M}$ is based on accumulated pre-clinical evidence [19]. The results of these studies proved that (a) siG12D$L O D E R^{T M}$ efficiently overcomes current siRNA delivery obstacles related to systemic delivery; (b) the LODER's pharmacokinetics (PK) enable dose reduction by orders of magnitudes and eliminates toxicity; (c) the strategy of local KRAS targeting by siG12D-LODER ${ }^{T M}$ can be effectively used to inhibit PDAC cell proliferation [19].

On the basis of these promising pre-clinical results, a first in-human Phase 1/2a clinical study of siG12D$L O D E R^{T M}$ in combination with chemotherapy was initiated for patients with locally advanced PDAC. The 
primary objectives were to assess safety and tolerability as well as to define a recommended phase $2 \mathrm{~b}$ dose (RP2D) of siG12D-LODER ${ }^{T M}$. Secondary objectives included measuring the efficacy potential of siG12D-LODER ${ }^{T M}$ (though limited by a single dosing design), the antitumor effects on the basis of tumor response, CA19-9 levels, Time To Metastasis (TTM), PFS and OS.

\section{RESULTS}

\section{LODER $^{T M}$ characteristics}

$L O D E R^{T M}$ is a miniature biodegradable matrix designed by Silenseed, allowing slow and prolonged local release of the encapsulated drug. $L O D E R^{T M}$ was developed to be inserted and placed into solid tumors using a standard biopsy procedure. siG12D-LODER ${ }^{T M}$ was designed to release anti-KRASG12D siRNA (siG12D) within the pancreatic tumor for four months. siG12D-LODER ${ }^{\mathrm{TM}}$ was shown to efficiently protect the encapsulated siRNA against enzymatic degradation, while releasing the drug within a tumor in vivo in mice (reference 19 and Figure 2A). The selected $L O D E R^{T M}$ matrix components are all FDA Generally Recognized As Safe (GRAS) materials, and the active agent siG12D was found to be non-toxic in all doses in vivo. Serial histopathological examination demonstrated that the drug covers the entire tumor mass typically within a week (Figure 2). Our data are consistent with previous evidence of uptake of naked siRNA within solid tumors [20]. Direct effects of KRAS expression inhibition on the mRNA and protein levels, as well as indirect effects of inhibition of tumor growth and induction of tumor cell death, were detected in the entire tumor mass (reference 19 and Figure 2B). Tumor growth was slowed as demonstrated in diverse in vivo mice models (subcutaneous and orthotopic), following implantation of siG12D-LODER $R^{T M}$ and the mice survival rates were significantly improved [19]. In siG12D-LODER ${ }^{T M}$-treated tumor tissue, apoptosis of tumor cells and tissue necrosis were found to be widespread after typically one week in the entire tumor (reference 19 and Figure 2B).

In addition to local effects on the tumor, we have shown in preclinical studies that siG12D slows tumor cell migration (Supplementary Figure 1C) and inhibits Epithelial to Mesenchymal Transition (EMT) (data not shown). Consistent with the above observations, we have found that mice treated with siG12D-LODERs did not develop metastases as compared with two thirds of untreated controls and empty-LODER ${ }^{T M}$-treated groups, which developed metastases (Supplementary Figure 1D).

The siG12D dosage in humans was selected based on the in vivo results in mice (Supplementary Figure 1A-1B). We have estimated a maximum dose of $3 \mathrm{mg}$ of siRNA above which RNAi saturation occurs.

siG12D-LODER ${ }^{T M}$ also affects the second most abundant KRAS mutation, G12V, which implies that the siG12D-LODER ${ }^{T M}$ efficacy is not restricted to the G12D mutation (data not shown). We further showed that siG12D provides additive benefit when used concomitantly with SOC chemotherapy, i.e. Gemcitabine or FOLFIRINOX (Supplementary Figure 2A-2B).

Systemically, the dose levels of the siG12D in the peripheral circulation are below detection levels, making measurements of (systemic) pharmacokinetics (PK) and pharmacodynamics (PD) impractical. However, the pharmacokinetics of siG12D drug in the tumor tissues can be assessed in vivo. We have studied PK and drug transport in a subcutaneous pancreatic tumor model. The amount of intercellular siG12D molecules that penetrate into cells was assessed by detection of antisense strand of the
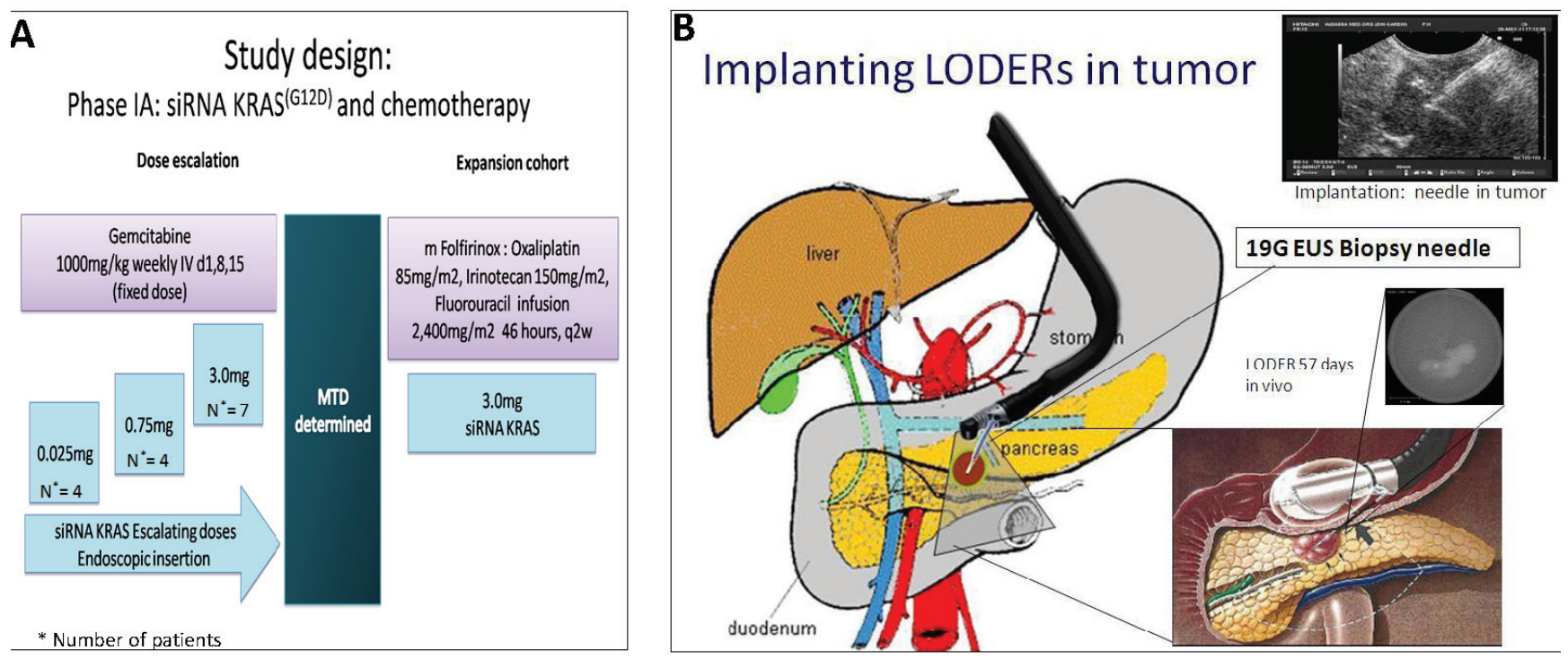

Figure 1: A. Study design. B. siG12D-LODER ${ }^{T M}$ is placed with Endoscopic US biopsy needle. 


\begin{tabular}{|c|c|c|c|c|}
\hline \multirow[b]{2}{*}{ Parameter } & \multicolumn{4}{|c|}{ siG12D LODER $R^{\text {m/ }}$ dose } \\
\hline & $\begin{array}{c}0.025 \mathrm{mg} \\
\mathrm{N}=4\end{array}$ & $\begin{array}{c}0.75 \mathrm{mg} \\
\mathrm{N}=4\end{array}$ & $\begin{array}{l}3 \mathrm{mg} \\
\mathrm{N}=7\end{array}$ & $\begin{array}{c}\text { All } \\
N=15\end{array}$ \\
\hline Mean Age $\pm \mathrm{SD}^{*}$, years & $65.8 \pm 12.5$ & $67.2 \pm 6.0$ & $71.5 \pm 8.9$ & $68.8 \pm 9.0$ \\
\hline Median, years & \multirow{2}{*}{$\begin{array}{c}64.4 \\
(52.1-82.0)\end{array}$} & 66.9 & 70.8 & 69.9 \\
\hline (Range) & & $(60.9-74.2)$ & $(57.6-85.3)$ & $(52.1-85.3)$ \\
\hline \multicolumn{5}{|l|}{ Gender: } \\
\hline Male, N (\%) & $3(75.0)$ & $1(25.0)$ & $4(57.1)$ & $8(53.3)$ \\
\hline Female, $\mathrm{N}(\%)$ & $1(25.0)$ & $3(75.0)$ & $3(42.9)$ & $7(46.7)$ \\
\hline \multicolumn{5}{|l|}{ Smoker: } \\
\hline Current (\%) & $1(25.0)$ & - & - & $1(6.7)$ \\
\hline Past (\%) & $1(25.0)$ & - & - & $1(6.7)$ \\
\hline \multicolumn{5}{|l|}{ Concomitant chemotherapy } \\
\hline \multirow[b]{2}{*}{ Treatment } & \multicolumn{4}{|c|}{ siG12D LODER ${ }^{T M}$ dose } \\
\hline & $\begin{array}{c}0.025 \mathrm{mg} \\
\mathrm{N}=4\end{array}$ & $\begin{array}{c}0.75 \mathrm{mg} \\
\mathrm{N}=4\end{array}$ & $\begin{array}{c}3 \mathrm{mg} \\
\mathrm{N}=7\end{array}$ & $\begin{array}{c}\text { All } \\
\mathrm{N}=15^{\star \star}\end{array}$ \\
\hline Gemcitabine & 2 & 4 & 3 & 9 \\
\hline $\begin{array}{l}\text { Qxaliplatin + Irinotecan } \\
\text { +Fluorouracil }\end{array}$ & - & - & 4 & 4 \\
\hline $\begin{array}{l}\text { Gemcitabine + Erlotinib + } \\
\text { Oxaliplatin }\end{array}$ & 1 & - & - & 1 \\
\hline None & 1 & - & - & 1 \\
\hline
\end{tabular}

A

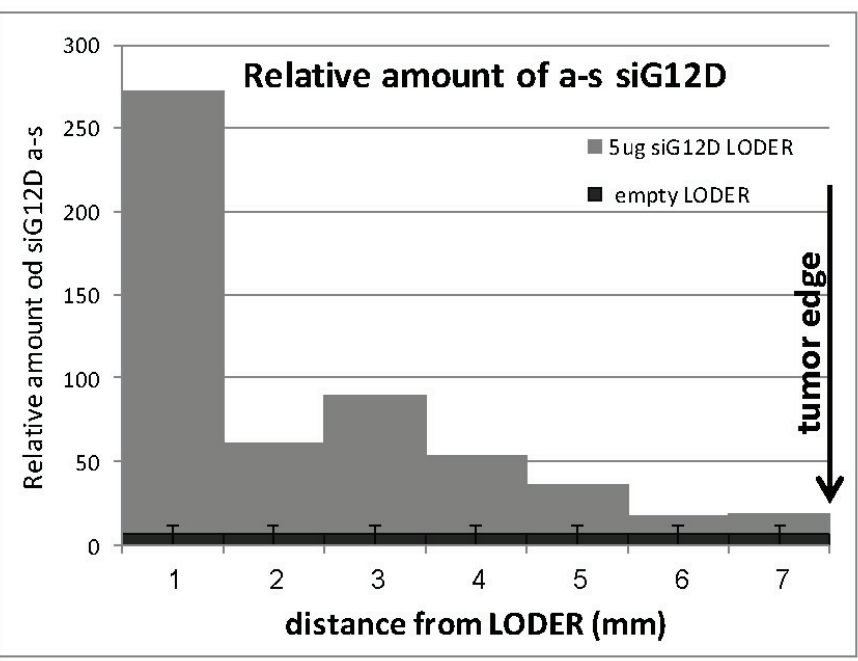

B

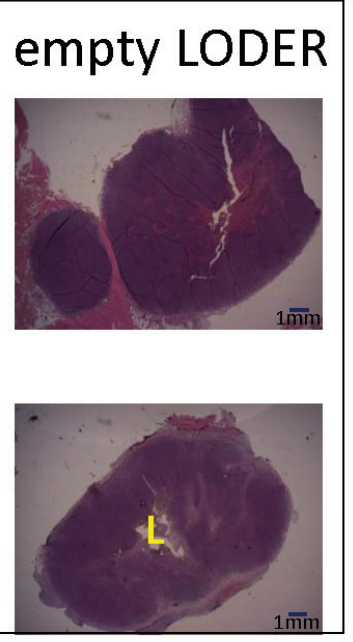

siG12DLODER
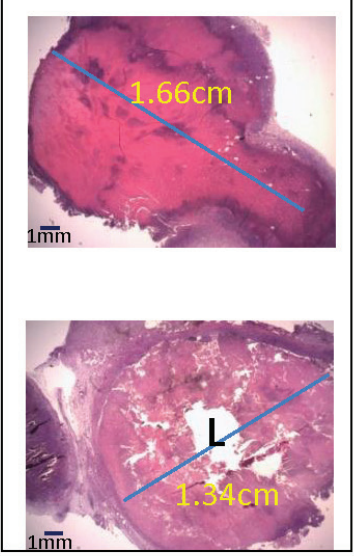

Figure 2: siG12D drug covers the entire tumor tissue within one week. Subcutaneous tumors of pancreatic PancO2 origin were treated with empty-LODER ${ }^{T M}$ or $L O D E R^{T M}$ containing $5 \mu \mathrm{g}$ siG12D. Seven days post-implantation mice were sacrificed, tumor tissue was formalin-fixed, paraffin embedded and cut to slices of $5 \mu \mathrm{m}$. A. The graph depicts relative amounts of antisense siG12D strand, measured by Relative Quantitative Real-Time PCR, at certain distances from the LODER ${ }^{T M}$ border. The results were normalized to RNU6 and calibrated to untreated control. B. Representative tumor tissue, H\&E stained, seven days post implantation. 
Table 2: Adverse events

summary of AEs occurring in $\geq 2$ patients in any treatment arm by preferred term

\begin{tabular}{|c|c|c|c|c|c|c|c|}
\hline & & $\begin{array}{r}0.025 \mathrm{mg} \\
\mathrm{N}=4\end{array}$ & & $\begin{array}{r}.75 \mathrm{mg} \\
\mathrm{N}=4\end{array}$ & $\begin{array}{r}3 \mathrm{mg} \\
\mathrm{N}=7\end{array}$ & & $\begin{array}{r}\text { All } \\
N=15\end{array}$ \\
\hline & $\mathbf{N}$ & $(\%)$ & $\mathbf{N}$ & $(\%)$ & & $\mathbf{N}$ & $(\%)$ \\
\hline Diarrhoea & 1 & $(25.0)$ & 1 & $(25.0)$ & & 7 & $(46.7)$ \\
\hline Abdominal pain & 1 & $(25.0)$ & 1 & $(25.0)$ & & 6 & $(40.0)$ \\
\hline Nausea & 1 & $(25.0)$ & 1 & $(25.0)$ & & 6 & $(40.0)$ \\
\hline Fatigue & - & - & 1 & $(25.0)$ & & 5 & $(33.3)$ \\
\hline Vomiting & - & - & 2 & $(50.0)$ & & 4 & (26.7) \\
\hline Pyrexia & 2 & $(50.0)$ & 1 & $(25.0)$ & & 3 & $(20.0)$ \\
\hline Rash & 1 & $(25.0)$ & 2 & $(50.0)$ & & 3 & $(20.0)$ \\
\hline Back pain & 1 & $(25.0)$ & - & - & & 3 & $(20.0)$ \\
\hline Anaemia & - & - & 2 & $(50.0)$ & & 2 & (13.3) \\
\hline Neutropenia & - & - & 2 & $(50.0)$ & & 2 & (13.3) \\
\hline Influenza like illness & - & - & - & - & & 2 & (13.3) \\
\hline Infusion related reaction & - & - & - & - & & 2 & (13.3) \\
\hline Platelet count decreased & - & - & - & - & & 2 & (13.3) \\
\hline Alopecia & - & - & - & - & & 2 & $(13.3)$ \\
\hline
\end{tabular}

Summary of procedure-related AEs

\begin{tabular}{|c|c|c|c|c|c|c|c|c|}
\hline \multirow{2}{*}{ Preferred term } & \multirow[t]{2}{*}{ Relationship } & \multicolumn{2}{|c|}{$\begin{array}{c}0.025 \mathrm{mg} \\
\mathrm{N}=4\end{array}$} & \multicolumn{2}{|c|}{$\begin{array}{c}0.75 \mathrm{mg} \\
\mathrm{N}=4\end{array}$} & \multicolumn{2}{|c|}{$\begin{array}{l}3 \mathrm{mg} \\
\mathrm{N}=7\end{array}$} & $\begin{array}{c}\text { All } \\
\mathrm{N}=15\end{array}$ \\
\hline & & $\mathbf{N}$ & $(\%)$ & $\mathbf{N}(\mathbf{O}$ & & & $(\%)$ & N $(\%)$ \\
\hline Pancytopenia & Possibly related & - & - & - & & 1 & (14.3) & $1 \quad(6.7)$ \\
\hline Abdominal pain & Possibly related & - & - & - & & 2 & $(28.6)$ & $2(13.3)$ \\
\hline Colonic obstruction & Possibly related & - & - & - & & 1 & $(14.3)$ & $1 \quad(6.7)$ \\
\hline Constipation & Possibly related & - & - & - & & 1 & $(14.3)$ & $(6.7)$ \\
\hline Pancreatitis & Possibly related & 1 & $(25.0)$ & - & & & - & $(6.7)$ \\
\hline Cholangitis & Definitely related & - & - & - & & & (14.3) & $(6.7)$ \\
\hline Renal failure & Possibly related & - & - & - & & 1 & (14.3) & $(6.7)$ \\
\hline
\end{tabular}

Summary of drug-related AEs

\begin{tabular}{|c|c|c|c|c|c|c|c|c|}
\hline \multirow[t]{2}{*}{ Preferred term } & \multirow[t]{2}{*}{ Relationship } & \multicolumn{2}{|c|}{$\begin{array}{c}0.025 \mathrm{mg} \\
\mathrm{N}=4\end{array}$} & \multicolumn{2}{|c|}{$\begin{array}{c}0.75 \mathrm{mg} \\
\mathrm{N}=4\end{array}$} & \multirow[t]{2}{*}{$\begin{array}{l}3 \mathrm{mg} \\
\mathrm{N}=7\end{array}$} & \multicolumn{2}{|c|}{$\begin{array}{c}\text { All } \\
\mathbf{N}=15\end{array}$} \\
\hline & & $\mathbf{N}$ & $(\%)$ & & $(\%)$ & & $\mathbf{N}$ & $(\%)$ \\
\hline Pancytopenia & Possibly related & - & - & - & - & $1(14.3)$ & 1 & $(6.7)$ \\
\hline Abdominal pain & Possibly related & - & - & - & - & $2(28.6)$ & 2 & $(13.3)$ \\
\hline Colonic obstruction & Possibly related & - & - & - & - & $1(14.3)$ & 1 & (6.7) \\
\hline Constipation & Possibly related & - & - & - & - & $1(14.3)$ & 1 & $(6.7)$ \\
\hline Renal failure & Possibly related & - & - & - & - & $1(14.3)$ & 1 & $(6.7)$ \\
\hline
\end{tabular}

* Abdominal pain possibly related to the procedure occurred in one patient. All other AEs occurred in one patient receiving FOLFIRINOX. In this patient, the AEs occurred 11 days after siG12D- LODERTM implantation or 5 days after initiation of FOLFIRINOX treatment and found by the DSMB to be unlikely related to the siRNA drug. 
molecule in the treated tissue using absolute quantitative Real-Time PCR. siG12D molecules were detected at distances extended by typical velocity of $1 \mathrm{~mm} /$ day until complete tumor coverage after a week. For example, in mouse subcutaneous model tumors of more than $1.7 \mathrm{~cm}$ in diameter were covered by siG12D molecule within a week (Figure 2A and data not shown). The PK results after a month are similar to the results after 7 days (data not shown), confirming a steady state of the drug distribution from $L O D E R^{T M}$. These direct measurements of the drug transport to extended distances are in line with results of the drug effect on the entire tissue. For example, Figure $2 \mathrm{~B}$ presents tumor tissue necrosis detected in the entire tumor area one week after siG12D-LODER ${ }^{T M}$ implantation (in this example, the tumor is extended to about $1.7 \mathrm{~cm}$ in diameter).

Shemi et al (in preparation, 2015) explores these results and their implications on drug transport in human solid tumors. They proposed a transport model which simulated drug delivery rates and demonstrated that drug delivery originating at the inner core of a tumor differs from systemic delivery. In systemic delivery drugs (or nanocarriers) need to cross the artery walls and penetrate into the inner tumor upstream, against the interstitial fluid pressure gradient. The transport of siRNA drug from the $L O D E R^{T M}$ surface into the entire tumor tissue was analysed and was explained by convection dominated transport (Péclet number $>1$, where the Péclet number reflects the rate of convection of a flow to its rate of diffusion). The drug is distributed from the inner core outwards in the tortuous tissue with effective velocity of $1 \mathrm{~mm} /$ day, and reaches the entire tumor mass in approximately one week.

\section{Patient characteristics}

The demographic and baseline characteristics of the study subjects in each treatment group are presented in Table 1. The median patient age was 70 years (range 52.185.3 ), where $30 \%$ of patients were above age of 74 . Fifteen patients with locally advanced PDAC were enrolled across three dose levels: $0.025 \mathrm{mg}, 0.75 \mathrm{mg}$ and $3.0 \mathrm{mg}$. Of these, four patients received a low dose $0.025 \mathrm{mg}$ of $s i G 12 D$ $L O D E R^{T M}$, four patients received $0.75 \mathrm{mg}$ (i.e. $0.375 \mathrm{mg} \mathrm{x} 2$ siG12D-LODER ${ }^{T M}$ ) and seven received $3 \mathrm{mg}$ (i.e. $0.375 \mathrm{mg}$ x 8 siG12D-LODER ${ }^{T M}$ ) (Figure 1A). Fourteen of the patients received concurrent $\mathrm{SOC}$ and one patient received no chemotherapy (Table 1). All 15 patients completed the study. Two patients were omitted from study but followed for safety due to metastatic disease detected on day one post siG12D-LODER ${ }^{T M}$ implant imaging. The average age of the study subjects at screening was $68.8 \pm 9.0$ years (range: 52.1-85.3). Eight subjects were males (53.3\%).

\section{Concomitant chemotherapy}

Nine patients received concomitant Gemcitabine as SOC $(50.0 \%$ of patients in the low treatment group, all patients in the mid-treatment group and $42.9 \%$ of patients in the high-treatment group). One patient received the Gemcitabine + Erlotinib + Oxaliplatin combination and one patient refused to receive any concomitant chemotherapy during the study. Four patients in the hightreatment (57.1\%) group received modified FOLFIRINOX (Table 1).
A

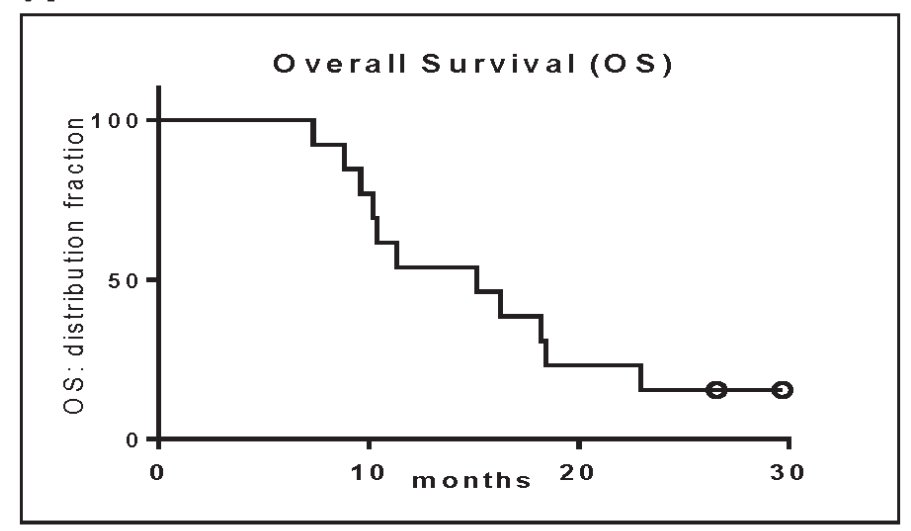

B

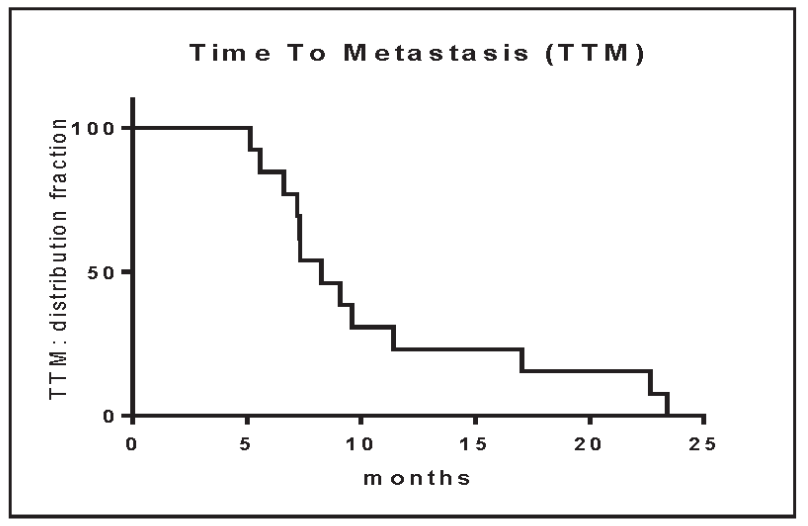

Legend: $-\bigcirc$ Product-Limit Estimate Curve

Figure 3: A. Overall survival: Kaplan-Meier curves depict OS of siG12D-LODER ${ }^{T M}$-treated patients. The two open circles mark patients who were still alive at the time of analysis. B. Time To Metastasis: Kaplan-Meier curves depict TTM of siG12D-LODER ${ }^{T M}$-treated patients. 


\section{Safety}

No DLTs (Dose Limiting Toxicity) events were observed during the study. No MTD (Maximal Tolerated Dose) was observed in the study. All 15 patients reported a total of 125 AEs. 111 of reported AEs (89\%) were grade 1 and 2, which were transient and resolved. The most common AEs (grade 1-2) were diarrhea and abdominal pain which were reported by seven patients $(46.7 \%)$, nausea and fatigue were reported by six (40\%) and five $(33.3 \%)$ patients respectively. 13 grade 3 and one grade 4 severe AEs were reported by $10(66.7 \%)$ subjects. The most common grade 3-4 AEs were neutropenia and cholangitis reported by 3 patients and 2 patients respectively.

AEs were separately analyzed as "procedurerelated" and "drug-related" (Table 2). Eight procedurerelated AEs (Table 2) were reported by four patients (26.7\%), of them, seven AEs were reported by three patients in the $3 \mathrm{mg}$ treatment group and one in the $0.025 \mathrm{mg}$ treatment group. Three procedure-related AEs in two patients were grade $1-2(38 \%)$. Only one AE (grade 3 cholangitis) was considered as definitely related to the study procedure, as it was observed one day after procedure. To note, the single grade $3 \mathrm{AE}$ related to pancreatitis, which was reported as 'possibly related' to the study procedure, seems to be unlikely related to procedure as it was identified 23 days after siG12D$L O D E R^{T M}$ implantation. In general, our experience both in animals [19] and humans is that there are no signs of pancreatitis. Even in cases where patients had previous resection (partial pancreatectomy), there is very rarely pancreatitis.

Six drug possibly-related AEs (Table 2) were reported by two patients $(14.3 \%)$ in the $3 \mathrm{mg}$ treatment group. In one patient the two reported AEs (abdominal pain and constipation) were of grade 1 and were defined one day after siG12D-LODER ${ }^{T M}$ implantation. Four remained drug possibly-related AEs were reported by one patient and occurred 11 days post siG12D-LODER ${ }^{T M}$ implantation and four days after initiating FOLFIRINOX. Of these, one was grade 2 renal failure. The remaining three AEs (grade 4 pancytopenia and grade 3 abdominal pain and colonic obstruction), were considered as possibly

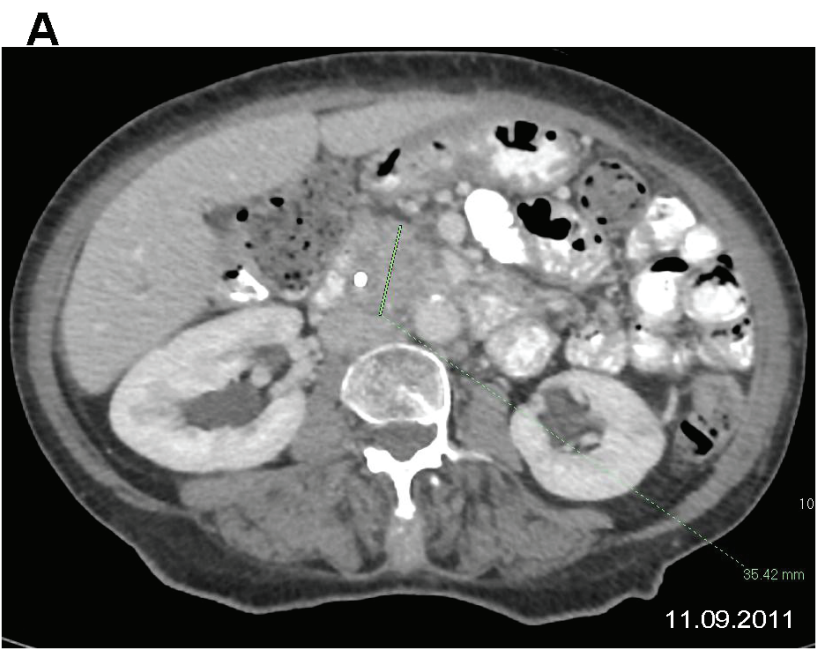

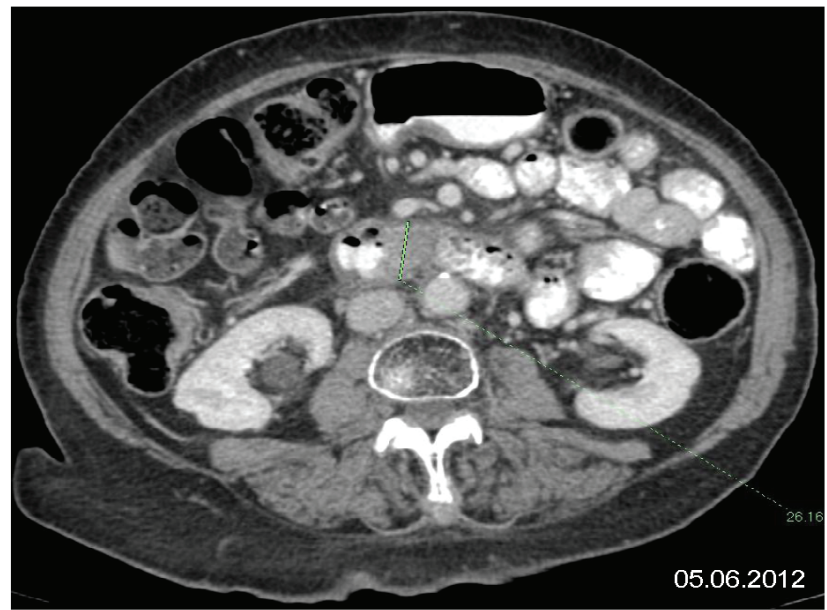

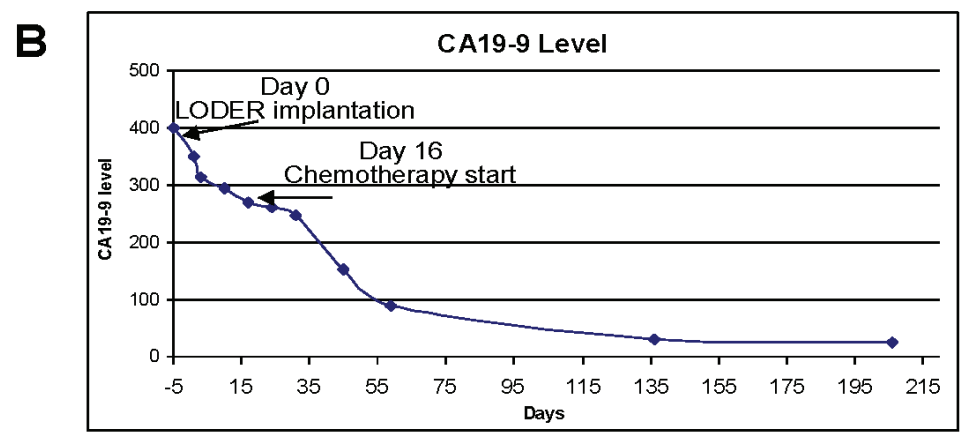

Figure 4: Anti-tumor effect of combination treatment with siG12D-LODER ${ }^{T M}$ in locally advanced non-operable PAC in a patient: A. left panel: a CT scan was performed prior to the implantation of siG12D-LODER ${ }^{T M}$ using EUS; tumor measures $35.42 \mathrm{~mm}$ in longest diameter; right panel: nine months later a significant tumor mass reduction is shown on a follow-up CT scan, tumor measures $26.16 \mathrm{~mm}$ in the longest diameter. B. The level of CA19-9 in blood, showing $23 \%$ decrease immediately after $L O D E R^{T M}$ insertion, prior SOC treatment. 
related to the study procedure and possibly related to the FOLFIRINOX chemotherapy, while are unlikely to be related to the siRNA drug itself, as concluded by the Data Safety Monitoring Board (DSMB). Overall, siG12D was safe and well tolerated in doses of up to $3 \mathrm{mg}$.

\section{Efficacy}

Efficacy results in this study are based on single dosing only (without repeat dosing after four months). Median OS for all patients was 15.12 months with $95 \%$ confidence intervals (CI) of 10.19 to 18.44 months. One year, 18 months and two years survival were $53.8 \%, 38.5 \%$ and $15.4 \%$ respectively. Data are based on predictive Kaplan-Meier analysis, valid to December 2014, where two (from Cohort III) out of 13 patients were still alive (Figure 3A). The first patient to die was after 7.36 months. Significant differences of OS between the three dose groups were not observed. Of note, in FOLFIRINOXtreated group $(n=3)$, median OS was longer than 27 months (while submitting this manuscript, two patients are alive, more than 27 and 30 months).

None of the patients showed tumor progression (PFS) at all analyzed time points (Figure 5).
The TTM was over 5.16 months in all patients. Median TTM was 8.25 months with $95 \%$ CI of 7.20 to 11.40 months. At 18 months TTM was $15.4 \%$. Data is based on predictive Kaplan-Meier analysis valid to December 2014, (Figure 3B). Significant differences of TTM between the three dose groups were not observed. In FOLFIRINOX-treated group, median TTM was 22.65 months.

In particular, we present a case of one of the patients from the low dose group. The patient initiated Gemcitabine chemotherapy 16 days after the siG12D$L O D E R^{T M}$ implantation. The treatment was well tolerated, with no significant side-effects. The serum-based tumormarker CA19-9 decreased by $22.4 \%$ following the siG12D-LODER ${ }^{T M}$ implantation before the administration of the first line chemotherapy treatment, and eventually reached normal values (Figure 4B). This patient then received local radiation treatment. A CT scan performed nine months post insertion of siG12D showed a significant decrease in tumor size (Figure 4A). This patient had 17.03 months TTM and died 18.21 months after enrollment to the study.
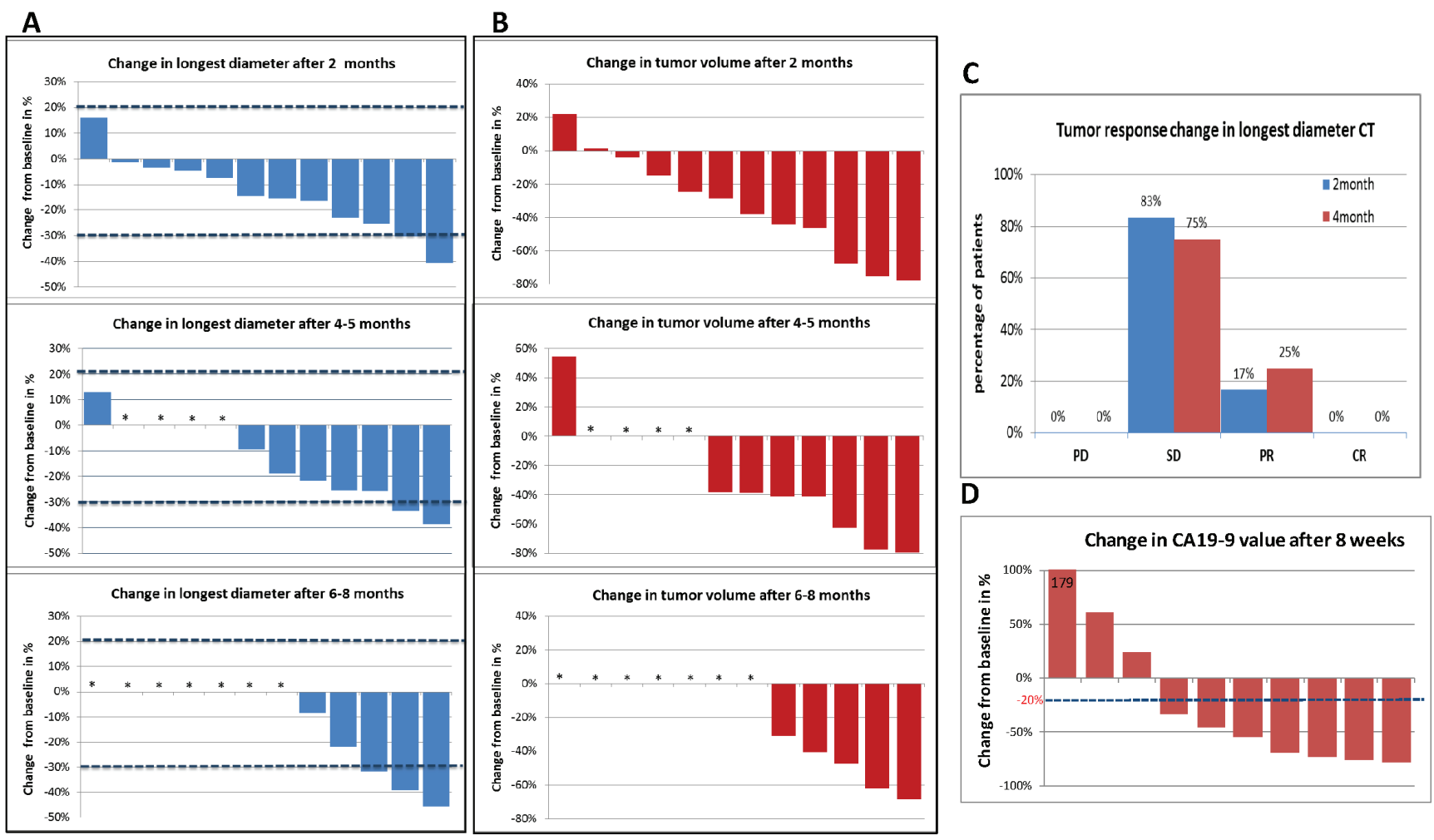

Figure 5: CT changes from base-line: The difference in the CT measurements shown as a waterfall plot. A. Change in longest diameter (LD) 2, 4 and 6-8 months after siG12D-LODER ${ }^{T M}$ implantation. ${ }^{*}$ ) marks the cases for which data after 4 months or later were not available. B. Change in tumor volume 2, 4 and 6-8 months after siG12D-LODER ${ }^{T M}$ implantation. (*) marks the cases for which data after 4 months or later were not available. C. Percentage of patients who showed progression of disease (PD); stable disease (SD); partial response (PR) or complete response (CR), based on the changes in LD according to the RECIST 1.1 guidelines. D. CA19-9 changes after 8 weeks: The graph shows waterfall plot of changes from baseline in the levels of the CA19-9 tumor marker. 


\section{CT analysis}

Figure $5 \mathrm{~A}$ and $5 \mathrm{~B}$ display $\mathrm{CT}$ response in the longest diameter (LD, based on direct measurements) and in volume (based on 3D reconstruction), respectively, of tumors in 12 patients at 2 months following the siG12D$L O D E R^{T M}$ insertion and after approximately four months and 6-8.5 months in patients were data was available. Measurements of changes in LD show that none of the tumors progressed according to RECIST1.1 (above $20 \%$ ); two patients showed decrease in LD below $30 \%$. Analysis at four months and at 6-8.5 months after the procedure showed that two of eight $(25 \%)$ and three of five $(60 \%)$ patients achieved partial response, respectively. Two patients (13.3\%) had stable disease (one each in the $0.75 \mathrm{mg}$ and the $3 \mathrm{mg}$ treatment groups). To note, none of the patient for whom CT data is available showed tumor progression $(\mathrm{LD}>20 \%)$ in the first 8 months $(\mathrm{PFS}=100 \%$ at the first $\sim 6-8$ months). Figure $5 \mathrm{~B}$ shows a significant decrease in tumor volume in most patients.

\section{Post-therapy CA19-9 changes}

At enrollment, abnormal elevated tumor marker CA19-9 levels ( $>37 \mathrm{U} / \mathrm{mi})$ were found in 10 patients among the 13 patients who were analyzed for efficacy. Decrease of $>20 \%$ is significant, following Ziske et al.'s report demonstrating that the decrease of CA19-9 $>20 \%$ after 8 weeks of chemotherapy is able to separate patients into groups with significantly different survival times [21]. In this study, decrease in tumor marker CA19-9 was observed in $70 \%$ of patients $(7 / 10)$. The data of 8 -weeks follow-up showed significant decrease in all the 7 patients (Figure 5D).

\section{DISCUSSION}

Pancreatic cancer is still one of the major challenges in clinical oncology. Mutant KRAS is a driving oncogene in the majority of human pancreatic cancer cases [14].

We have met this challenge by developing a therapeutic platform for local and prolonged delivery of siRNA. Our report describes an efficient, clinically applicable siRNA delivery method that overcomes the major obstacles of toxicity and organ accessibility. Notably, our approach enabled the conversion of KRAS from a non-druggable to a potentially druggable cancer target.

The present study evaluated single administration of three escalating doses of siG12D together with standard of care chemotherapy in patients with locally advanced inoperable pancreatic cancer. The primary endpoints were to evaluate the safety and toxicity of siG12D in combination with chemotherapy. No DLTs were observed during the study, therefore no MTD could be determined, and the highest dose administered and well tolerated was $3 \mathrm{mg}$. Most AEs were grade 1 and 2, transient, and not related to the study drug or to the study procedure. In the $3 \mathrm{mg}$ treatment group, the AEs were related to either the implantation procedure, or the chemotherapy treatment (FOLFIRINOX).

The results of the Phase 1/2a study demonstrated a median OS of 15.12 months, a median TTM of 8.25 months; 18 months OS was $38.5 \%$ and TTM $15.4 \%$. Most patients had stable disease and two patients had demonstrated partial responses. Six to 8.5 months after $L O D E R^{T M}$ insertion, $60 \%$ of the patients of whom CT data is available achieved partial response, and $40 \%$ had stable disease. No dose response was observed between the dose of siG12D and OS or TTM. Of note, two patients from the high dose group are still alive (27 and 30 months).

In this study we present the affectivity of LODER ${ }^{T M}$ for RNAi based treatment for solid tumors. Our strategy combines selection of cancer-driver target with prolonged delivery to cancer cells. The LODER ${ }^{T M}$ platform is based on encompassing of siRNA within a miniature biodegradable polymeric matrix that protects and enables the release of the siRNA drug for an extended duration of time regionally within tumor tissue. The LODER ${ }^{T M}$ is specifically designed to be inserted with existing EUS biopsy procedures. We showed the efficacy of siG12D drug, and that the innovative siG12D-LODER ${ }^{T M}$ delivery system is efficient, site-specific, and safe.

In summary, the concomitant administration of siG12D-LODER ${ }^{T M}$ and chemotherapy was safe and well tolerated. The prolonged clinical benefit warrants further evaluation of this agent in combination with chemotherapy. A multinational randomized Phase $2 \mathrm{~b}$ clinical trial is currently in progress (NCT01676259).

\section{PATIENTS, MATERIALS AND METHODS}

The Phase 1/2a study (NCT01188785) was conducted at three medical centers in Israel, namely the Chaim Sheba Medical Center, Tel HaShomer, the Hebrew University-Hadassah Medical Center, Ein Kererm Jerusalem, and the Shaare Zedek Hospital, Jerusalem. The siG12D-LODER ${ }^{\mathrm{TM}}$ polymeric implants were provided by Silenseed Ltd. Patients were enrolled between July, 2010 and May, 2012 in accordance with the principles of Good Clinical Practice and the Declaration of Helsinki. The protocol was approved by the Israeli Ministry of Health and Local Institutional Review Boards, and patients provided written informed consent before participation.

\section{Study population}

Patients with unresectable, locally advanced confirmed or highly suspected adenocarcinoma of the pancreas, or resectable tumors in patients who defer 
surgery due to a high surgical risk (e.g. coagulopathy or severe congestive heart failure) were enrolled. No upper age limit was applied. Eligibility criteria included patients eligible to receive SOC as first line treatment in accordance with treating physician recommendation, target tumor that was accessible for intra-tumoral administration by EUS guidance as determined by the radiologist/ gastroenterologist performing the EUS insertion, Karnofsky performance status of $\geq 70 \%$, life expectancy of $\geq 3$ months, serum creatinine $<2.0 \mathrm{mg} / \mathrm{dL}$, PT - INR $<1.5$, absolute neutrophil count $(\mathrm{ANC})>1,000 \times 10^{3}$ cells $/ \mathrm{mL}$, platelets $\geq 75,000 / \mathrm{mL}$, hemoglobin $\geq 10 \mathrm{mg} /$ $\mathrm{dL}$, no other malignancy present that would interfere with the current intervention and have measurable disease. Patients were excluded if they had distant metastases, including liver, lung, or lymph nodes, peritoneal spread or malignant ascites. Other exclusion criteria included clinically significant pancreatitis within 12 weeks of treatment, medical condition that would preclude both percutaneous and endoscopic guided delivery of the any concurrent medical illness or medical condition that would compromise patient safety or the objectives of the study as determined by a study investigator, or history of bleeding coagulopathy.

\section{Study design}

This study was an open label, escalating single dose Phase $1 / 2$ a multi-center trial. Men and women $\geq 18$ years old diagnosed with non-operable PDAC or with high evidence of PDAC with no evidence of distant metastasis were screened for eligibility. Metastases presence was assessed by CT scan. Adenocarcinoma of pancreas was confirmed by biopsy.

In this study, single dosing (single time) of escalating doses of $\operatorname{siG} 12 D-\operatorname{LODER}^{T M}(0.025,0.75$ and $3 \mathrm{mg})$ were administered by a standard procedure EUS guidance. siG12D-LODERs were inserted into the tumor using the FDA approved equipment, namely EchoTip ${ }^{\circledR}$ Ultra (Cook Medical) or the equivalent Expect 19 Flex Biopsy needle (Boston Scientific) (Figure 1). After insertion patients were monitored carefully for signs of systemic or local treatment-related toxicity. Scheduled physical and clinical examinations, vital signs (estimated by Karnofsky performance status (PS)), laboratory tests, tumor marker CA19-9, abdominal CT and disease assessments were performed during the course of the trial. Following the siG12D-LODER ${ }^{T M}$ insertion Gemcitabine $1000 \mathrm{mg} / \mathrm{m}^{2} \mathrm{IV}$ was given on a weekly basis until disease progression. One patient received Gemcitabine + Erlotinib + Oxaliplatin $\left(1000 \mathrm{mg} / \mathrm{m}^{2}, 100 \mathrm{mg}\right.$ and $100 \mathrm{mg} / \mathrm{m}^{2} \mathrm{IV}$ respectively) for 3 weeks, followed by Gemcitabine alone, $1000 \mathrm{mg} / \mathrm{m}^{2}$ IV on a weekly basis. The RP2D (recommended Phase $2 \mathrm{~b}$ dose) was further examined in highest dose cohort in combination with modified FOLFIRINOX (Oxaliplatin $85 \mathrm{mg} / \mathrm{m}^{2}$, Irinotecan $150 \mathrm{mg} / \mathrm{m}^{2}$ followed by a Fluorouracil continuous IV infusion of $2,400 \mathrm{mg} / \mathrm{m}^{2} 46$ hours every two weeks). Active follow-up period was eight weeks after placement of siG12D-LODER ${ }^{T M}$ and long-term survival follow-up was performed until death. After two months, CT scan was performed as a SOC at each study site; the results were presented in a blinded to the sponsor for analysis.

\section{Safety assessments}

Toxicity was evaluated according to the NCI Common Terminology Criteria for Adverse Events (CTCAE), Version 4.0. A minimum of four patients were entered at each dose level. All four subjects were followed until they completed the cycle of therapy and subsequent enrolment of new cohorts was based on the toxicity assessment in that first cycle and the documentation of any dose limiting toxicities. A two week gap was enforced between cohorts. Before opening the next higher dose level, all toxic effects at the preceding dose level were reviewed and expansion or escalation was undertaken as appropriate. Meetings between investigators were organized as required.

\section{Efficacy measurements}

Antitumor activity was assessed based on changes in CA19-9 and imaging. Changes were based on RECIST 1.1 criteria. Imaging was performed at baseline and at 12 weeks, and in most patients subsequently after 4 months and 6 months. Progression free survival (PFS) was defined based on the CT data (based on RECIST1.1) as the first time to show longest diameter (LD) $>20 \%$. Overall survival (OS) was defined from the date of diagnosis until death from any cause. TTM was defined from the date of diagnosis until detection of metastasis. Data is based on predictive Kaplan Meier analysis valid until December 2014, when two (from Cohort III) out of 13 patients were still alive, and one having not demonstrated disease progression (after 27 months).

\section{Statistical analysis}

All measured variables and derived parameters were listed individually and, if appropriate, tabulated by descriptive statistics. Descriptive statistics and summary tables were provided giving sample size (n), absolute and relative frequency of categorical variables and sample size, arithmetic mean, standard deviation, coefficient of variation (if appropriate), median, minimum and maximum percentiles and $95 \%$ confidence interval (CI) for means of continuous variables. All two-tailed statistical tests with a $P \leq 0.05$ were considered significant. All adverse events were classified by System Organ Class and 
preferred terminology according to MedDRA dictionary and were summarized in cross tables by severity and dose group. All tests applied were two-tailed, and $P$ values $\leq$ 0.05 were considered significant. The data were analyzed using the $\mathrm{SAS}^{\circledR}$ software version 9.1 (SAS Institute, Cary, North Carolina).

All authors had access to the study data, and reviewed and approved the final manuscript.

\section{ACKNOWLEDGMENTS}

The authors would like to thank Yechiel Goldman for valuable editorial assistance.

\section{CONFLICTS OF INTEREST}

There is no conflict of interest

\section{REFERENCES}

1. Delpu Y, Hanoun N, Lulka H, et al. Genetic and epigenetic alterations in pancreatic carcinogenesis. Curr Genomics 2011;12:15-24.

2. Von Hoff DD, Ervin T, Arena FP, et al. Increased survival in pancreatic cancer with nab-paclitaxel plus gemcitabine. N Engl J Med 2013;369:1691-703.

3. Conroy T, Desseigne F, Ychou M, et al. FOLFIRINOX versus gemcitabine for metastatic pancreatic cancer. N Engl J Med 2011;364:1817-25.

4. Iacobuzio-Donahue $\mathrm{CA}, \mathrm{Fu} \mathrm{B}$, Yachida $\mathrm{S}$, et al. DPC4 gene status of the primary carcinoma correlates with patterns of failure in patients with pancreatic cancer. J Clin Oncol 2009;27:1806-13.

5. Chauffert B, Mornex F, Bonnetain F, et al. Phase III trial comparing intensive induction chemoradiotherapy (60 Gy, infusional 5-FU and intermittent cisplatin) followed by maintenance gemcitabine with gemcitabine alone for locally advanced unresectable pancreatic cancer. Definitive results of the 2000-01 FFCD/SFRO study. Ann Oncol 2008;19:1592-9.

6. Loehrer PJ, Sr., Feng Y, Cardenes H, et al. Gemcitabine alone versus gemcitabine plus radiotherapy in patients with locally advanced pancreatic cancer: an Eastern Cooperative Oncology Group trial. J Clin Oncol 2011;29:4105-12.

7. Herman JM, Wild AT, Wang H, et al. Randomized Phase III Multi-Institutional Study of TNFerade Biologic With Fluorouracil and Radiotherapy for Locally Advanced Pancreatic Cancer: Final Results. J Clin Oncol 2013;31:88694.

8. Blazer M, Wu C, Goldberg RM, et al. Neoadjuvant Modified (m) FOLFIRINOX for Locally Advanced Unresectable (LAPC) and Borderline Resectable (BRPC) Adenocarcinoma of the Pancreas. Ann Surg Oncol 2014.

9. Belda-Iniesta C, Ibanez de Caceres I, Barriuso J, et al.
Molecular biology of pancreatic cancer. Clin Transl Oncol 2008;10:530-7.

10. Jones S, Zhang X, Parsons DW, et al. Core signaling pathways in human pancreatic cancers revealed by global genomic analyses. Science 2008;321:1801-6.

11. Feldmann G, Beaty R, Hruban RH, et al. Molecular genetics of pancreatic intraepithelial neoplasia. J Hepatobiliary Pancreat Surg 2007;14:224-32.

12. Villanueva A, Reyes G, Cuatrecasas M, et al. Diagnostic utility of K-ras mutations in fine-needle aspirates of pancreatic masses. Gastroenterology 1996;110:1587-94.

13. Sun C, Yamato T, Furukawa T, et al. Characterization of the mutations of the K-ras, p53, p16, and SMAD4 genes in 15 human pancreatic cancer cell lines. Oncol Rep 2001;8:8992.

14. Singh A, Greninger P, Rhodes D, et al. A gene expression signature associated with "K-Ras addiction" reveals regulators of EMT and tumor cell survival. Cancer Cell 2009; 15:489-500.

15. John J, Sohmen R, Feuerstein J, et al. Kinetics of interaction of nucleotides with nucleotide-free H-ras p21. Biochemistry 1990;29:6058-65.

16. Wu SY, Lopez-Berestein G, Calin GA, et al. RNAi therapies: drugging the undruggable. Sci Transl Med 2014;6:240ps7.

17. Fleming JB, Shen GL, Holloway SE, et al. Molecular consequences of silencing mutant K-ras in pancreatic cancer cells: justification for K-ras-directed therapy. Mol Cancer Res 2005;3:413-23.

18. Rejiba S, Wack S, Aprahamian M, et al. K-ras oncogene silencing strategy reduces tumor growth and enhances gemcitabine chemotherapy efficacy for pancreatic cancer treatment. Cancer Sci 2007;98:1128-36.

19. Zorde Khvalevsky E, Gabai R, Rachmut IH, et al. Mutant KRAS is a druggable target for pancreatic cancer. Proc Natl Acad Sci U S A 2013;110:20723-8.

20. Kim SH, Jeong JH, Lee SH, et al. Local and systemic delivery of VEGF siRNA using polyelectrolyte complex micelles for effective treatment of cancer. J Control Release 2008;129:107-16.

21. Ziske C, Schlie C, Gorschluter M, et al. Prognostic value of CA 19-9 levels in patients with inoperable adenocarcinoma of the pancreas treated with gemcitabine. $\mathrm{Br} \mathrm{J}$ Cancer 2003;89:1413-7. 